\title{
Y-Shaped, Self-Expanding Metallic Stents (SEMS) to Drain Malignant Strictures of the Liver Hilum: Y Are They Better?
}

\author{
John Baillie ${ }^{1} \cdot$ Ravi Vachhani ${ }^{1}$
}

Published online: 16 November 2016

(c) Springer Science+Business Media New York 2016

In the last 20 years, the prognosis of hilar cholangiocarcinoma (aka Klatskin tumor) has progressed from almost certain death to a potentially survivable lesion, thanks to advances in surgical technique, portal vein embolization, chemoradiation, and liver transplantation. Undoubtedly, the revised anatomic classification of these lesions based on vascular supply (the Bismuth classification) "jumpstarted" this revolution [1]. In the era dominated by a palliative approach, endoscopists were largely focused on how best to achieve relief of jaundice and itching, often a major misery for these unfortunate patients. The magnitude and quality-of-life impact of pruritus due to obstructive jaundice should never be underestimated. One of us (JB) recalls a patient with malignant biliary strictures who needed to regularly roll on a rough coconut doormat in the hospital as the principal means of relieving his dreadful itching.

The debate about unilateral versus bilateral biliary drainage has continued for many years, with the pendulum swinging back and forth. Certainly, inconvenience and discomfort are increased by the presence of two percutaneous biliary drains, in particular when one might suffice. In addition to discomfort and leakage, they limit mobility and in some cases deplete patients of fluid and electrolytes. A very few patients who have external biliary drains have been reported to develop a brisk choleresis with literally liters of bile filling their catheter bags [2]. This creates the potential for acute kidney injury from intravascular volume depletion. Due to these preventable adverse effects,

John Baillie

john.baillie@vcuhealth.org

1 Gastroenterology, Hepatology and Nutrition, Virginia Commonwealth University, Richmond, VA 23298, USA patients with percutaneous drains for biliary obstruction are currently closely monitored for fluid and electrolyte balance (especially sodium, potassium, and bicarbonate) accompanied by daily decision making regarding their early removal.

In this issue of the Digestive Diseases and Sciences, Jiao et al. [3], from Zhengzhou, China, described their initial experience with a newly designed, Y-configured, bilateral self-expanding metallic stent (SEMS) for the treatment of hilar biliary obstruction. In a retrospective review of 14 patients, mainly with cholangiocarcinoma, but also with gallbladder cancer, this novel, Y-shaped, uncovered SEMS was placed across a hilar stricture. The procedure is arduous: It requires bilateral percutaneous transhepatic biliary access, starting with right- and left-sided drainage catheters, percutaneous balloon dilation (over a guide wire) of the malignant stricture, followed by serial placement of the metallic stents (SEMS) in two pieces, one L-shaped, the other (shorter) "limb" aligning with radio-dense (gold) markers to create a Y-shaped device. The authors state that this approach is superior to existing methods such as stentthrough-stent and side-by-side. In a mean study period of 298 days (range 89-465 days), 6/14 patients developed stent occlusion, all but one due to tumor ingrowth, the longest stent patency (281 days) in comparison with 15 prior studies of bilateral biliary drainage using Y-shaped stents. Patency rates at 6, 9, and 12 months were 92, 63, and $32 \%$, respectively. During the mean stent patency period (281 days), 9 patients (64\%) died, 8 from multiple organ failure due to tumor progression, and one from myocardial infarction. By Kaplan-Meier analysis, median patient survival was $381 \pm 45.7$ days, with survival at 6,9 , and 12 months being 92, 84, and $32 \%$, respectively.

Do these data indicate that Jiao et al. have "cracked" this problem, or do their superior results merely reflect 
selection bias? The answer to this question is further obscured by the observation that almost half of the subjects in this retrospective trial received chemotherapy or radiation for their cancers. The initial success rate for stent placement exceeded $90 \%$, with only a single failure. Satisfactory bile drainage was evidenced by a significant fall of serum bilirubin concentration. In one case, attributed to rapid progression of the tumor, the bilirubin remained elevated requiring maintenance of percutaneous access. Early ( $<30$ day) complications were individual instances of severe hemobilia and acute cholangitis, whereas late complications were cholangitis and cholecystitis. The reported hemobilia was due to a pseudoaneurysm of the hepatic arterial supply that was managed by angiography and subsequent coil embolism. Stent occlusion-when it occurred-was managed by placing a second uncovered SEMS through the novel device.

The authors acknowledge that a significant limitation of their study was the small number of patients enrolled $(n=14)$. Patients were considered eligible if they were jaundiced, with histologically proven malignant hilar obstruction, who had refused surgery, or were considered unresectable or inoperable with patient ages ranging from 49 to 81 years. Exclusions from the study, typical for stent recipients, included patients with severe coagulopathy, refractory ascites, partial or complete portal vein thrombosis, and/or Child's class $\mathrm{C}$ status. Of those whose stents occluded, two declined further procedures and died 40 and 55 days later. Those who agreed to further intervention were kept alive longer, although one patient required continuous external drainage due to duodenal obstruction, and another received a unilateral plastic stent instead of a SEMS.

How should this small, retrospective study with seemingly optimistic results be regarded? What factors influenced the eventual outcome? None of these patients underwent liver transplantation. In the setting of primary sclerosing cholangitis (PSC) with the development of cholangiocarcinoma, transplantation has undoubtedly provided a therapeutic option where previously there was none. Although Gores et al. [4] cite a 65-70\% 5-year survival after successful liver transplantation, $30 \%$ died during the first year on the waiting list with a recurrence rate of $20 \%$, suggesting that brachytherapy followed by liver transplantation may be more effective than standard chemoradiation in this setting [5]. What about unilateral versus bilateral biliary drainage? Although the rationale is that the undrained lobe or segments of the liver will atrophy and no longer present problems, little data support this approach. The Asia-Pacific Working Group on Hepatobiliary Cancer recommends draining $>50 \%$ of the liver volume to preserve functional volume during chemotherapy and to prevent procedure-related cholangitis arising in an undrained liver lobe [6], consistent with the appealing logic that the liver should be maximally drained. Although the detailed description of the placement of biliary catheters and subsequent deployment of the Y-shaped stent is beyond the scope of this commentary, suffice it to say, the technical aspects are quite demanding: the authors deserve our admiration for their perseverance especially in light of the considerable trial-and-error necessary to perfect the process. The results were impressive; at least until tumor ingrowth blocked the lumen in almost half the cases, which could possibly be delayed by placement of a partially coated SEMS.

First, computed tomography (CT) and percutaneous transhepatic cholangiography (PTC) are performed, with percutaneous biopsy to confirm histology followed by placement of bilateral biliary drains. Five-seven days later, the stenosis is balloon dilated, with placement of the Y-shaped stent, followed by insertion of two 8.5 Fr drainage catheters. After a further 7-14 days, cholangiography is repeated and the drains removed if the Y-shaped stent is patent. In the case of rapid tumor progression, or continued high-grade stenosis despite balloon dilation, external drainage has to be retained, usually requiring at least 2-3 weeks before the Y-shaped stent can function adequately. The authors did not consider leakage around catheters and pain at the insertion site to be significant issues, which likely reflects the stoicism of Chinese population studied.

This study is a welcome addition to the growing literature on Y-shaped SEMS in the management of malignancy involving the liver hilum [7, 8]. The authors of the current study opine that a prospective, randomized trial is now needed to confirm their findings; we have to agree. Although a small, retrospective study like this is likely riddled with confounding issues, it offers a tantalizing look at what may be possible in well-selected candidates. Nevertheless, the community of advanced gastrointestinal endoscopists is probably close to, or has already reached the limits of what can be achieved by effective biliary drainage alone. Solid tumors of the liver hilum still have a fearsome reputation; increased survival will now have to depend on novel cancer therapies. Masters of Molecular Medicine, please to step up to the hilum [9]!

\section{References}

1. Bismuth H, Collette MB. Intrahepatic cholangioenteric anastomosis in carcinoma of the hilus of the liver. Surg Gynecol Obstet. 1975; 140:170-178.

2. Sandborn WJ, Gross JB Jr., Larson DE, et al. High-volume postobstructive choleresis after transhepatic external biliary drainage resolves with conversion to internal drainage. J Clin Gastroenterol. 1993;17:42-45. 
3. Jiao D, Huang K, Zhu M, et al. Placement of a newly designed, Y-configured, bilateral self-expanding metallic stent for hilar biliary obstruction: a pilot study. Dig Dis Sci. (Epub ahead of print). doi:10.1007/s10620-016-4284-1.

4. fGores GJ, Murad D, Heimback JK, Rosen CB. Liver transplantation for perihilar cholangiocarcinoma. Dig Dis. 2013;31:126-129.

5. Mukewar S, Gupta A, Baron TH, et al. Endoscopically-inserted nasobiliary catheters for high dose-rate brachytherapy as part of neoadjuvant therapy for perihilar cholangiocarcinoma. Endoscopy. 2015;47:878-883.

6. Rerknimitr R, Angsuwatcharakon P, Ratanachu-ek T, et al. AsiaPacific consensus recommendations for endoscopic and interventional management of hilar cholangiocarcinoma. J Gastroenterol Hepatol. 2013;28:593-607.

7. Chahal P, Baron TH. Expandable metal stents for endoscopic stent-within-stent placement for malignant hilar biliary obstruction. Gastrointest Endosc. 2010;71:195-199.

8. Srinivasan I, Kaheleh M. Metal stents for hilar lesions. Gastrointest Endosc Clin N Am. 2012;22:555-565.

9. Forsmark CE, Diniz AL, Zhu AX. Consensus conference on hilar cholangiocarcinoma. HBP (Oxford). 2015;17:666-668. 